\title{
The Trigonometric Parallax of Cygnus X-1
}

\section{Citation}

Reid, Mark J., Jeffrey E. McClintock, Ramesh Narayan, Lijun Gou, Ronald A. Remillard, and Jerome A. Orosz. 2011. "The Trigonometric Parallax of Cygnus X-1." The Astrophysical Journal 742, no. 2: 83.

\section{Published Version}

doi:10.1088/0004-637X/742/2/83

\section{Permanent link}

http://nrs.harvard.edu/urn-3:HUL.InstRepos:13041330

\section{Terms of Use}

This article was downloaded from Harvard University's DASH repository, and is made available under the terms and conditions applicable to Other Posted Material, as set forth at http:// nrs.harvard.edu/urn-3:HUL.InstRepos:dash.current.terms-of-use\#LAA

\section{Share Your Story}

The Harvard community has made this article openly available.

Please share how this access benefits you. Submit a story.

Accessibility 


\title{
THE TRIGONOMETRIC PARALLAX OF CYGNUS X-1
}

\author{
Mark J. Reid ${ }^{1}$, Jeffrey E. McClintock ${ }^{1}$, Ramesh Narayan ${ }^{1}$, Lijun Gou ${ }^{1}$, Ronald A. Remillard ${ }^{2}$, and Jerome A. Orosz ${ }^{3}$ \\ ${ }^{1}$ Harvard-Smithsonian Center for Astrophysics, 60 Garden Street, Cambridge, MA 02138, USA \\ ${ }^{2}$ Kavli Institute for Astrophysics and Space Research, Massachusetts Institute of Technology, 70 Vassar Street, Cambridge, MA 02139, USA \\ ${ }^{3}$ Department of Astronomy, San Diego State University, 5500 Campanile Drive, San Diego, CA 92182, USA \\ Received 2011 June 17; accepted 2011 July 19; published 2011 November 9
}

\begin{abstract}
We report a direct and accurate measurement of the distance to the X-ray binary Cygnus X-1, which contains the first black hole to be discovered. The distance of $1.86_{-0.11}^{+0.12} \mathrm{kpc}$ was obtained from a trigonometric parallax measurement using the Very Long Baseline Array. The position measurements are also sensitive to the 5.6 day binary orbit and we determine the orbit to be clockwise on the sky. We also measured the proper motion of Cygnus $\mathrm{X}-1$ which, when coupled to the distance and Doppler shift, gives the three-dimensional space motion of the system. When corrected for differential Galactic rotation, the non-circular (peculiar) motion of the binary is only about $21 \mathrm{~km} \mathrm{~s}^{-1}$, indicating that the binary did not experience a large "kick" at formation.
\end{abstract}

Key words: astrometry - black hole physics - stars: distances - stars: individual (Cygnus X-1) - X-rays: binaries

Online-only material: color figures

\section{INTRODUCTION}

In this paper (Paper I), we resolve the long-standing problem of the distance to Cygnus X-1 by measuring its trigonometric parallax, i.e., triangulating using the Earth's orbit as one leg of the triangle and measuring the change in its apparent position as the Earth orbits the Sun. Our results are based on observations of compact radio emission with the National Radio Astronomy Observatory's ${ }^{4}$ (NRAO's) Very Long Baseline Array (VLBA). Previously, only one trigonometric parallax has been published for a binary containing a black hole (BH): V404 Cyg, an X-ray binary with a low-mass $\left(M<1 M_{\odot}\right)$ companion star (MillerJones et al. 2009). Our result is the first trigonometric parallax for an X-ray binary with a massive companion $\left(M \gtrsim 10 M_{\odot}\right)$; an accurate distance is key to achieving secure and detailed models of the physical properties of the binary system.

Cygnus $\mathrm{X}-1$ was the first $\mathrm{BH}$ candidate to be established via dynamical observations (Webster \& Murdin 1972; Bolton 1972). It is one of the most well-studied BH systems with excellent optical and X-ray data. However, modeling this system is limited by its very uncertain distance. Distance estimates span a very large range, with most values between 1.8 and $2.4 \mathrm{kpc}$ (Ziolkowski 2005). The Cygnus X-1 distance estimate with the smallest formal uncertainty is $2.14 \pm 0.07 \mathrm{kpc}$ by Massey et al. (1995) and is based on photometry and spectroscopy of NGC 6871 (an open cluster in the Cyg OB3 complex). While this distance estimate is very precise, its true accuracy (including systematic sources of error involving extinction, metallicity corrections, and stellar modeling) is perhaps $\pm 15 \%$. Also, it (and many other distance estimates) relies on the association of Cygnus X-1 with the Cyg OB3 complex. While such an association is supported by similar stellar (Hipparcos) and Cygnus X-1 (VLBI) proper motion measurements (Lestrade et al. 1999; Mirabel \& Rodrigues 2003), it still must be considered tentative as this line of sight through the Milky Way is very "crowded," passing nearly parallel to the Local spiral arm, which extends at least several kpc from the Sun, and then

\footnotetext{
4 The National Radio Astronomy Observatory is a facility of the National Science Foundation operated under cooperative agreement by Associated Universities, Inc.
}

through the Perseus and Outer arms at greater distances (Reid et al. 2009b).

\section{OBSERVATIONS AND DATA ANALYSIS}

Our observations were conducted under VLBA program BR141. We observed Cygnus X-1 and two background sources over $10 \mathrm{hr}$ tracks at five epochs: 2009 January 23, April 13, July 13, and October 31, and 2010 January 25. These dates well sample the peaks of the sinusoidal trigonometric parallax signature in both right ascension and declination. This sampling provides near maximum sensitivity for parallax detection and ensures that we can separate the secular proper motion (caused by projections of Galactic rotation as well as any peculiar motion of Cygnus X-1 and the Sun) from the sinusoidal parallax effect. Table 1 lists the positions of Cygnus X-1 and the background continuum sources, as well as their angular separations from Cygnus X-1.

Generally, data calibration followed similar procedures as for parallax observations of continuum sources in the Orion nebular cluster at $8.4 \mathrm{GHz}$ (Menten et al. 2007). We placed observations of well-known strong sources near the beginning, middle, and end of the observations in order to monitor delay and electronic phase differences among the intermediate frequency bands. In practice, however, we found minimal drifts and used only a single scan of J2005+7752 for this calibration.

In order to calibrate atmospheric propagation delays, we scheduled "geodetic blocks" consisting of typically a dozen ICRF sources (Fey et al. 2004) observed over a wide range of source azimuths and elevations as described in Reid et al. (2009a). We observed in left circular polarization and spread eight $16 \mathrm{MHz}$ bands so as to span $\approx 500 \mathrm{MHz}$ of bandwidth. We corrected the interferometer data for ionospheric delays using global models of the total electron content obtained from global positioning system observations (Walker \& Chatterjee 2000). Residual multi-band delays and fringe rates were measured and used as data to solve for clock offsets and drift rates and unmodeled zenith atmospheric propagation path delays. Later these were removed from the rapid-switching (parallax) data.

The rapid-switching observations employed four adjacent frequency bands of $16 \mathrm{MHz}$ bandwidth and recorded both right 

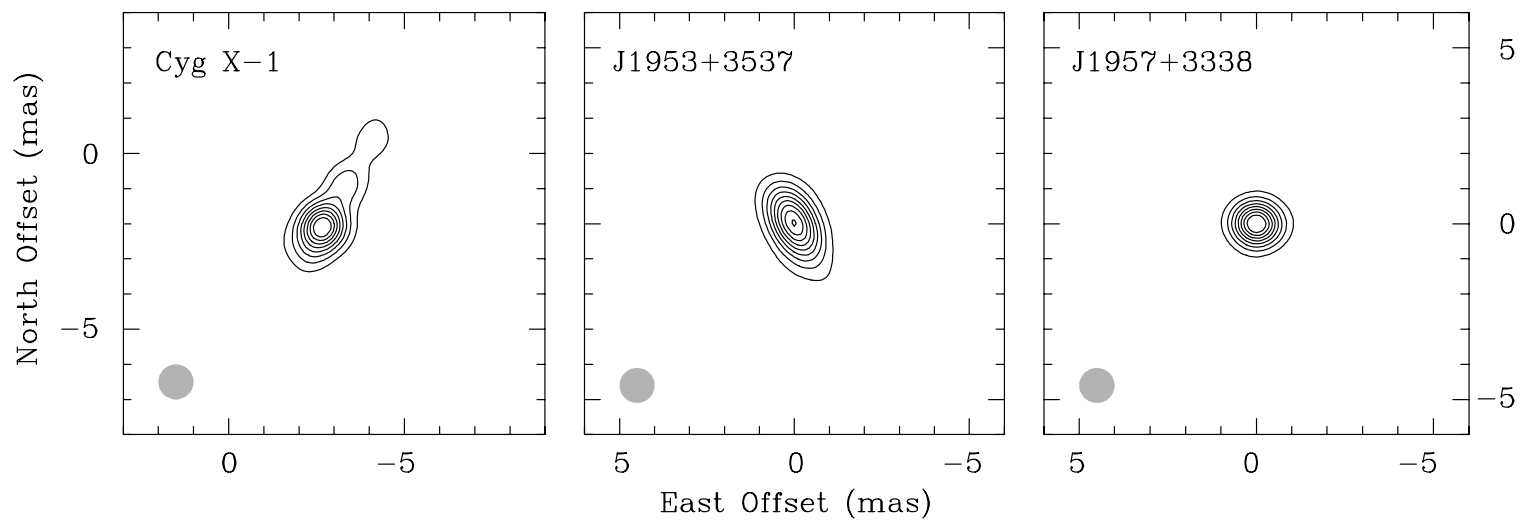

Figure 1. Images of Cygnus X-1 and the two background continuum sources from the middle epoch (2009 July 13). Source names are in the upper left corner and restoring beams are in the lower left corner of each panel. All contour levels are integer multiples of $1 \mathrm{mJy} \mathrm{beam}^{-1}$ for Cygnus X-1 and $15 \mathrm{mJy}^{\mathrm{b}}$ beam ${ }^{-1}$ for the background sources. Zero contours are suppressed for clarity; negative brightness levels were below the first contour level for all images.

Table 1

Source Positions

\begin{tabular}{lcccc}
\hline \hline Source & $\begin{array}{c}\text { R.A. (J2000) } \\
(\mathrm{h} \mathrm{m} \mathrm{s})\end{array}$ & $\begin{array}{c}\text { Decl. (J2000) } \\
\left(\text { days }^{\prime \prime \prime}\right)\end{array}$ & $\begin{array}{c}\theta_{\text {sep }} \\
(\text { deg })\end{array}$ & $\begin{array}{c}\text { P.A. } \\
(\text { deg })\end{array}$ \\
\hline Cygnus X-1 & 195821.672762 & 351205.72511 & $\ldots$ & $\ldots$ \\
J1953+3537 & 195330.875712 & 353759.35927 & 1.1 & -66 \\
J1957+3338 & 195740.549923 & 333827.94339 & 1.6 & -175 \\
\hline
\end{tabular}

Notes. The position for Cygnus X-1 at $8.45 \mathrm{GHz}$ comes from the parallax fitting at epoch 2009.570. This position is relative to the unweighted average of the two background sources, which gave coordinate differences of about \pm 0.25 mas, consistent with their ICRF catalog uncertainties of 0.5 mas (Fey et al. 2004). Angular offsets $\left(\theta_{\text {sep }}\right)$ and position angles (P.A.) east of north relative to Cygnus $\mathrm{X}-1$ are indicated in Columns 4 and 5 .

and left circularly polarized signals. The four (dual-polarized) bands were centered at frequencies of $8.425,8.441,8.457$, and $8.473 \mathrm{GHz}$. We alternated between two $\approx 11$ minute blocks, each consisting of observations of Cygnus X-1 and one of the background sources. Within a block, we switched sources every $40 \mathrm{~s}$, typically achieving $30 \mathrm{~s}$ of on-source data. We used the background sources J1953+3537 and J1957+3338 for phasereferencing.

We calibrated the correlated data using the NRAO Astronomical Image Processing System (AIPS). The calibration sequence included four steps. The first step involved correction of interferometer delays and phases for the effects of diurnal feed rotation (parallactic angle), for errors in the values of Earth's orientation parameters used at the time of correlation, and for any small position shifts in the a priori coordinates of either the target or background sources. Since the VLBA correlator model includes no ionospheric delays, we used global total electron content models to remove ionospheric effects. At this point, we also corrected the data for residual zenith atmospheric delays and clock drifts (determined from the geodetic block data).

In the second step, we adjusted interferometer visibility amplitudes for the small (few \%) effects of biases in the threshold levels of the data samplers at each station. We also entered system temperature and antenna gain curve information into calibration tables, which allows conversion of correlation coefficients to flux density units. In the third step, we performed a "manual phase-calibration" to remove delay and phase differences among all eight $16 \mathrm{MHz}$ bands. This was done by selecting one scan on a strong calibrator, fitting fringe patterns to the data for each frequency band, and then shifting delays and phases to remove offsets. The fourth calibration step involved determining antenna-based complex gains from each of the two background sources and interpolating and removing those from the Cygnus X-1 observations. For most antennas at most times the phases were easily "connected." However, when interferometer phase differences between adjacent background source scans exceeded $60^{\circ}$, the data between those times were discarded.

We then imaged the calibrated data with the AIPS task IMAGR. The synthesized "dirty" beam typically had an FWHM of $1.55 \times 0.86$ mas elongated approximately in the north-south direction. We adopted a round convolving beam of 1.0 mas to construct CLEAN images. Example images from the middle epoch are shown in Figure 1. Positions were determined by fitting elliptical Gaussian brightness distributions to the images. For Cygnus X-1, which shows a core-jet structure, we restricted the fitted pixels to those close to the bright core, typically within 1 mas of the peak emission. The peak brightness of Cygnus X-1 ranged between 4 and $9 \mathrm{mJy}_{\text {beam }}^{-1}$ among our observations.

\section{PARALLAX AND PROPER MOTION FITTING}

The change in position of Cygnus $\mathrm{X}-1$ relative to a background continuum source was then modeled by the parallax sinusoid in both coordinates, completely determined by one parameter (the parallax), and a secular proper motion in each coordinate (see Figure 2). The model included the effects of the ellipticity of Earth's orbit. The weighting of the data in the parallax and proper motion fitting is complicated because the formal position uncertainties are often unrealistically small, since a priori unknown sources of systematic error often dominate over random noise. The north-south components of relative positions often have greater uncertainty than the east-west components because the interferometer beams are generally larger in the north-south direction and systematic errors from unmodeled atmospheric delays usually are more strongly correlated with north-south positions (Honma et al. 2008).

In order to allow for, and estimate the magnitude of, systematic errors, we assigned independent "error floors" to the east-west and north-south position data and added these floors in quadrature with the formal position-fitting uncertainties. Trial parallax and proper motion fits were conducted and a reduced $\chi_{v}^{2}$ (per degree of freedom) statistic was calculated separately for the east-west and north-south residuals. The error floors were then adjusted iteratively so as to make $\chi_{v}^{2} \approx 1.0$ for each coordinate. This procedure resulted in error floors of 0.08 and 

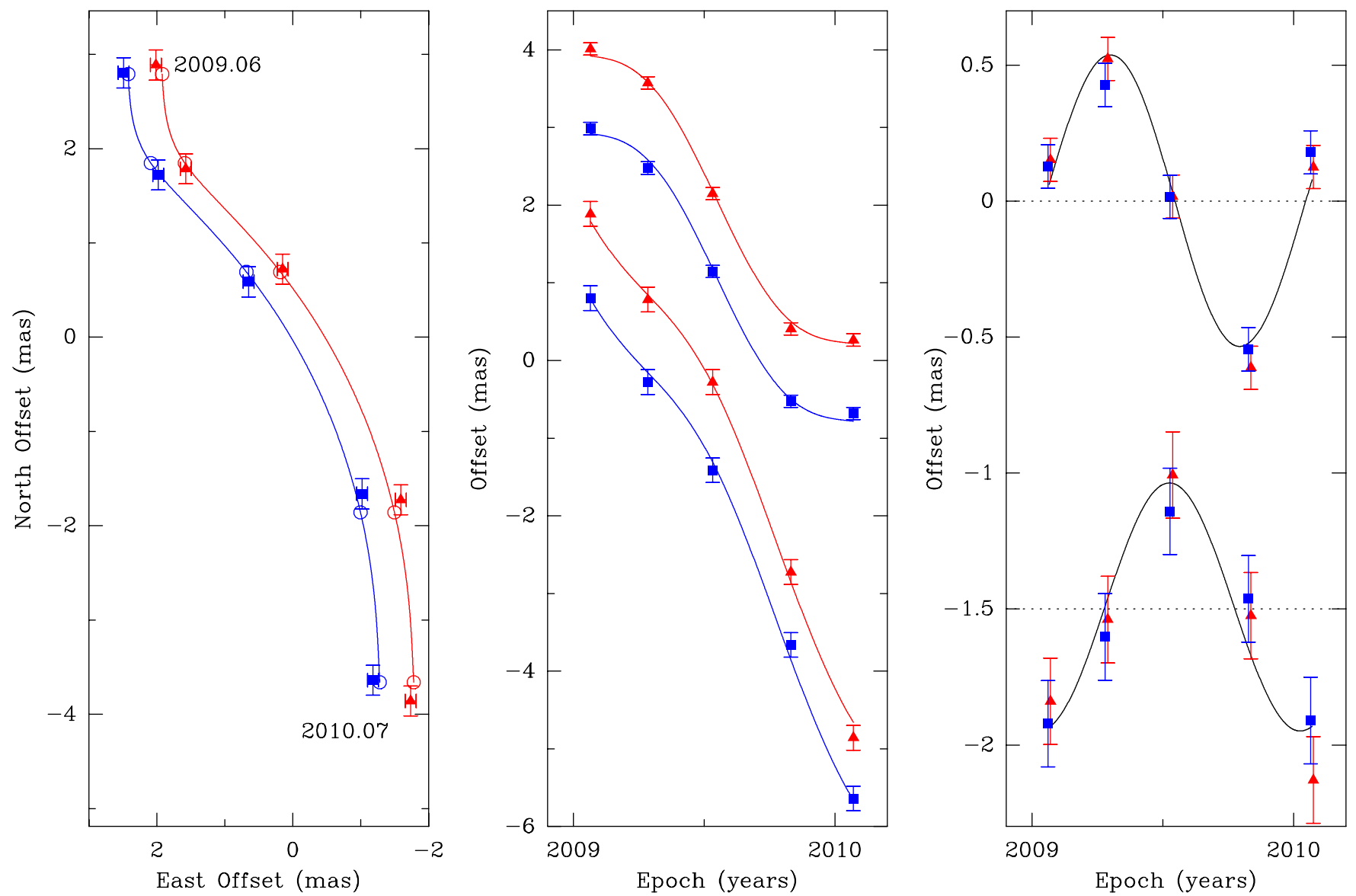

Figure 2. Parallax and proper motion data and fits. Plotted are position offsets of Cygnus X-1 relative to the two background sources: J1953+3537 (red triangles) and J1957+3338 (blue squares). The degree-scale separations of the absolute positions given in Table 1 have been removed. The yearly sinusoidal position variations (the parallax signature) caused apparent position shifts of Cygnus X-1 as the Earth orbits the Sun. Left panel: positions on the sky with first and last epochs labeled. Data for the two background source are offset horizontally for clarity. The expected positions from the parallax and proper motion fit are indicated (circles). Middle panel: east (top lines) and north (bottom lines) position offsets and best-fit parallax and proper motions vs. time. Data for the two background sources and two coordinates are offset vertically for clarity. Right panel: same as the middle panel, except the best-fit proper motions have been removed, allowing all data to be overlaid and the effects of only the parallax seen. The north offset data have been offset vertically (below) the east offset data for clarity.

(A color version of this figure is available in the online journal.)

Table 2

Cygnus X-1 Parallax and Proper Motion Fits

\begin{tabular}{|c|c|c|c|c|c|c|}
\hline Cygnus X-1 Orbit & $\begin{array}{l}\text { Background } \\
\text { Source }\end{array}$ & $\begin{array}{c}\text { Parallax } \\
\text { (mas) }\end{array}$ & $\begin{array}{c}\mu_{x} \\
\left(\text { mas y }^{-1}\right)\end{array}$ & $\begin{array}{c}\mu_{y} \\
\left(\text { mas }^{-1}\right)\end{array}$ & $\chi^{2}$ & $\bar{N}$ \\
\hline None & $\begin{array}{c}\mathrm{J} 1953+3537 \\
\mathrm{~J} 1957+3338 \\
\text { Combined }\end{array}$ & $\begin{array}{l}0.582 \pm 0.055 \\
0.511 \pm 0.061 \\
0.547 \pm 0.041\end{array}$ & $\begin{array}{l}-3.74 \pm 0.10 \\
-3.66 \pm 0.11 \\
-3.70 \pm 0.08\end{array}$ & $\begin{array}{l}-6.56 \pm 0.19 \\
-6.28 \pm 0.21 \\
-6.42 \pm 0.14\end{array}$ & $\begin{array}{r}4.57 \\
5.54 \\
12.82\end{array}$ & $\begin{array}{r}5 \\
5 \\
13\end{array}$ \\
\hline $\begin{array}{l}\mathrm{CCW} \\
\mathrm{CW}\end{array}$ & $\begin{array}{l}\text { Combined } \\
\text { Combined }\end{array}$ & $\begin{array}{l}0.528 \pm 0.047 \\
0.539+0.033\end{array}$ & $\begin{array}{l}-3.68 \pm 0.09 \\
-3.78+0.06\end{array}$ & $\begin{array}{l}-6.36 \pm 0.17 \\
-6.40 \pm 0.12\end{array}$ & $\begin{array}{r}16.81 \\
8.49\end{array}$ & $\begin{array}{l}12 \\
12\end{array}$ \\
\hline
\end{tabular}

Notes. Combined fits used a common parallax and proper motion parameters for both background sources. The proper motion components are defined as $\mu_{x}=\mu_{\alpha} \cos \delta$ and $\mu_{y}=\mu_{\delta}$, where $\alpha$ is right ascension and $\delta$ is declination. The binary orbital model assumed an orbital period of 5.599829 days, superior conjunction at JD 2441874.71 days, a radius of the radio source about the center of mass of 0.071 mas, an inclination of $36^{\circ}$, and spin-axis of $-26^{\circ}$ east of north. We tried two orbital directions: clockwise (CW) and counterclockwise (CCW) on the sky. Columns 6 and 7 list the post-fit $\chi^{2}$ values and the number of degrees of freedom $(N)$. Parameter uncertainties have been re-scaled to those appropriate for a $\chi_{v}^{2}$ (per degree of freedom) of unity.

0.16 mas for the eastward and northward position measurements, respectively. The magnitudes of these error floors are reasonably consistent with those obtained for other parallax targets observed at $8.4 \mathrm{GHz}$, e.g., Menten et al. (2007), and are probably dominated by unmodeled ionospheric delays. Any component of variability in the centroid of the core-jet position of Cygnus X-1 caused by changing jet opacity must be less than $\approx 0.1$ mas.

The fitting results are presented in Table 2. First, we attempted separate parallax fits for each background source measurement. The parallaxes for Cygnus X-1 for the two background sources differ by only 0.071 mas and are consistent with their individual 


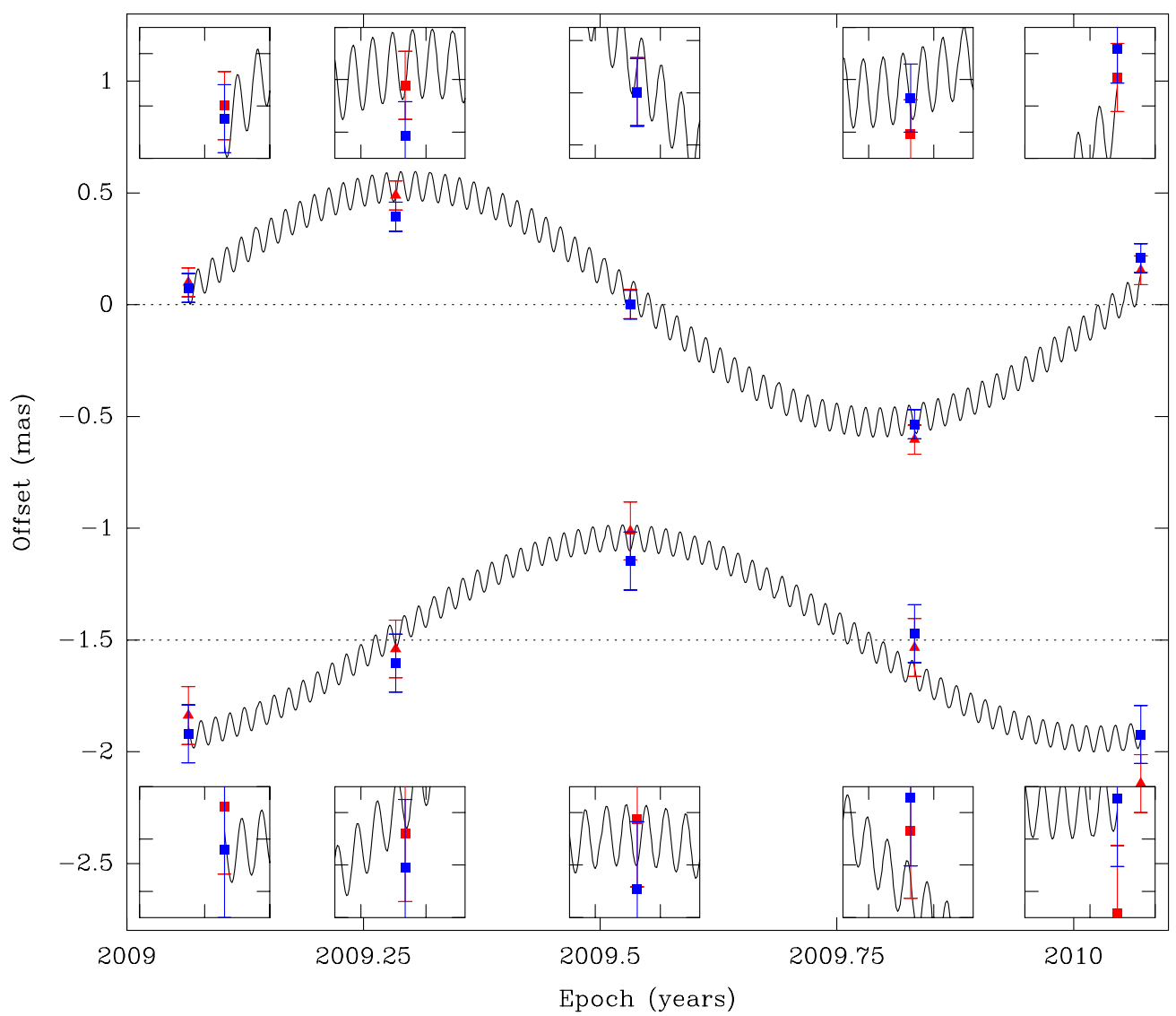

Figure 3. Parallax fit including the effects of a clockwise (on the sky) binary orbit for the radio source relative to the center of mass of Cygnus X-1. East (top) and north (bottom) position offsets and best-fitting model (lines) vs. time. Plotted are the positions of Cygnus X-1 relative to the two background sources: J1953+3537 (red triangles) and J1957+3338 (blue squares). Clearly seen are the annual parallax sinusoidal signature (with the proper motion removed) and the "high-frequency" oscillations caused by the 5.6 day orbit of the radio source (the black hole) about center of mass of the binary system. Blow-ups of the eastward (top small boxes) and northward (bottom small boxes) offsets each cover 0.1 year in time and 0.25 mas in offset.

(A color version of this figure is available in the online journal.)

formal uncertainties of about 0.06 mas. This agreement suggests that neither atmospheric mismodeling nor potential unresolved structural variations in the background sources contribute position errors greater than the error floors.

Next, because of the agreement of the Cygnus X-1 parallaxes and proper motions for the two background sources, we did a combined fit with common parallax and proper motion parameters for both sets of data. (In the combined fit, we did allow for different position offsets for Cygnus X-1 with respect to each background source, since the a priori positions for these sources were not known to better than $\approx 0.5$ mas.)

Finally, we investigated the effect of the Cygnus X-1 binary orbit on the parallax fits. All orbital parameters are known with reasonable accuracy and were considered independent variables (Brocksopp et al. 1999; Gies et al. 2008; Orosz et al. 2011) with the exception of the direction of the orbital rotation on the sky. The binary orbital model we adopted had an orbital period of 5.599829 days, superior conjunction at JD 2441874.707 days, a radius of the radio source about the center of mass of $0.128 \mathrm{AU}$, an inclination of $36^{\circ}$ (i.e., the angle between the orbit angular momentum vector and our line of sight). (Using the revised value of $i=27^{\circ}$, determined in Paper II, results in no significant difference in parallax and proper motion fits.) We assumed an orbital angular momentum vector parallel to the radio jet in Cygnus X-1, observed in our images (see Figure 1) and previous studies (Stirling et al. 2001) to be about $-26^{\circ}$ east of north. This leaves two possible orbital directions, clockwise $(\mathrm{CW})$ and counterclockwise $(\mathrm{CCW})$ on the sky, to be determined by the data.

Parallax and proper motion fits for both orbital directions are listed in Table 2. The $\chi^{2}$ value for the CW orbit was 8.49 (for 13 degrees of freedom), which was significantly lower than the value of 16.81 for the CCW orbit. The agreement of the data with the CW orbit model can be seen in the position data in Figure 3, especially in the more accurate eastward offset data. Thus, our astrometric observations appear to be sensitive to the position changes caused by orbit of the radio source (presumably the $\mathrm{BH}$ ) about the center of mass of the binary system, and we have directly determined the direction of the orbit to be $\mathrm{CW}$ on the sky.

Adopting the $\mathrm{CW}$ orbit, we re-fitted the data allowing the orbital radius to vary and found a radius of $0.18 \pm 0.09 \mathrm{AU}$. Thus, the current VLBA data independently give a $2 \sigma$ estimate of the radius of the compact radio source about the center of mass of the binary. With more observations in the future, we could refine the values of some of the Cygnus X-1 orbital parameters.

We adopted a Cygnus X-1 parallax of $0.539 \pm 0.033$ mas from the fit with the lowest $\chi_{v}^{2}$ value. That fit combined the data from both background sources and used the orbital model with clockwise projected motion on the sky. This parallax for Cygnus X-1 corresponds to a distance of $1.86_{-0.11}^{+0.12} \mathrm{kpc}$. At this distance, the proper motion of Cygnus X-1 corresponds to $-33 \mathrm{~km} \mathrm{~s}^{-1}$ and $-56 \mathrm{~km} \mathrm{~s}^{-1}$ eastward and northward, respectively. Completing the full space velocity, the average 
heliocentric radial velocity of Cygnus $\mathrm{X}-1$ is about $-5 \mathrm{~km} \mathrm{~s}^{-1}$ (Gies et al. 2008).

\section{DISCUSSION}

Since we have measured the position, parallax, and proper motion, and others have measured the line-of-sight velocity, we have full three-dimensional position and velocity information for Cygnus X-1. We can convert its velocity vector from the equatorial heliocentric reference frame in which it is measured into a Galactic reference frame. A convenient Galactic frame is one rotating with a circular velocity $\Theta(R)$ at the position of the source: i.e., a "local standard of rest" at the location of Cygnus $\mathrm{X}-1$. This transformation is described in detail in Reid et al. (2009b).

Conversion to a rotating Galactic reference frame depends somewhat upon the solar motion and Galactic parameters. We adopt the distance of the Sun from the Galactic center to be $R_{0}=8.25 \mathrm{kpc}$ as a rounded average of the values quoted by Reid (1993) for a variety of "classical" techniques and by Ghez et al. (2008) and Gillessen et al. (2009) from the orbits of stars about the supermassive $\mathrm{BH}$ at the Galactic center. The rotation speed of the local standard of rest is $\Theta_{0} \approx 240 \mathrm{~km} \mathrm{~s}^{-1}$, which is based on this distance and the proper motion of Sgr A* (the supermassive $\mathrm{BH}$ at the Galactic center), which directly measures $\left(\Theta_{0}+V_{\odot}\right) / R_{0}=30.2 \mathrm{~km} \mathrm{~s}^{-1} \mathrm{kpc}^{-1}$ (Reid \& Brunthaler 2004). Using the newly proposed solar motion of $V_{\odot} \approx 11 \mathrm{~km} \mathrm{~s}^{-1}$ by Schönrich et al. (2010) and assuming a locally flat rotation curve, i.e., $\Theta(R)=\Theta_{0}$, we find a "peculiar motion" (relative to circular motion) for Cygnus X-1 of $17 \pm 1 \mathrm{~km} \mathrm{~s}^{-1}$ toward the Galactic center, $-11 \pm 3 \mathrm{~km} \mathrm{~s}^{-1}$ in the direction of Galactic rotation and $6 \pm 1 \mathrm{~km} \mathrm{~s}^{-1}$ toward the north Galactic pole. Thus, the total magnitude of the peculiar motion of Cygnus X-1 is only $21 \mathrm{~km} \mathrm{~s}^{-1}$, indicating that the binary system did not experience a large "kick" when the $\mathrm{BH}$ formed. This result supports the conjecture that the $\mathrm{BH}$ may have formed without a supernova explosion (Katz 1975; Mirabel \& Rodrigues 2003).

Recently, Xiang et al. (2011) modeled the scattering by interstellar dust grains of X-rays from Cygnus X-1. They considered 18 models describing the characteristics of dust and selected six models with iron column densities consistent with a value of $1.62 \times 10^{17} \mathrm{~cm}^{-2}$ (Lee et al. 2009). Modeling the $\mathrm{X}$-ray light curves, three of the six models yielded distances between 3.15 and $3.59 \mathrm{kpc}$, and these models were discarded as being incompatible with our trigonometric parallax. The remaining three models yielded distances between 1.72 and $1.90 \mathrm{kpc}$, compatible with our parallax. Thus, having an accurate trigonometic parallax can be important for the study of X-ray scattering from time varying sources.

The BH in Cygnus X-1 is fed gas via the stellar wind emanating from its O-type companion, which underfills its Roche equipotential lobe. The degree to which the star underfills its
Roche lobe must be tightly constrained in order to obtain a useful dynamical model of the system, and accurate knowledge of the source distance is necessary to accomplish this (Orosz et al. 2007). Using the accurate distance, $D$, reported here, we have constructed such a model and obtained precise values for the mass, $M$, of the $\mathrm{BH}$, the orbital inclination angle, $i$, and other system parameters, as reported in Paper II (Orosz et al. 2011). With the required, accurate values of $M, i$, and $D$ in hand, in Paper III we proceed (Gou et al. 2011) to model the X-ray continuum spectrum and determine the spin of the $\mathrm{BH}$. BH spin can be determined because the measured X-ray spectrum from the accretion disk is dominated by radiation coming from very near the BH. The temperature and luminosity of the X-ray emission increase greatly with increasing $\mathrm{BH}$ spin, as the inner radius of the accretion disk, $R_{\text {in }}$, shrinks toward the $\mathrm{BH}$ event horizon. $R_{\text {in }}$ can be determined by fitting the X-ray continuum emission from the BH's accretion disk to a fully relativistic model if the $\mathrm{BH}$ mass, distance, and accretion disk inclination angle are known (Li et al. 2005). Because an astronomical BH (which is believed to have negligible net electric charge) is completely described by specifying its mass and spin, our measurements of these quantities provide a complete description of the $\mathrm{BH}$ in Cygnus X-1.

Facility: VLBA

\section{REFERENCES}

Bolton, C. T. 1972, Nature, 235, 271

Brocksopp, C., Fender, R. P., Larionov, V., et al. 1999, MNRAS, 309, 1063

Fey, A. L., Ma, C., Arias, E. F., et al. 2004, AJ, 127, 3587

Ghez, A. M., Salim, S., Weinberg, N. N., et al. 2008, ApJ, 689, 1044

Gies, D. R., Bolton, C. T., Blake, R. M., et al. 2008, ApJ, 678, 1237

Gillessen, S., Eisenhauer, F., Trippe, S., et al. 2009, ApJ, 692, 1075

Gou, L., McClintock, J. E., Reid, M. J., et al. 2011, ApJ, 742, 85 (Paper III)

Honma, M., Tamura, Y., \& Reid, M. J. 2008, PASJ, 60, 951

Katz, J. I. 1975, Nature, 253, 698

Lee, J. C., Smith, R. K., Canizares, C. R., et al. 2009, arXiv:0902.4671

Lestrade, J.-F., Preston, R. A., Jones, D. L., et al. 1999, A\&A, 344, 1014

Li, L.-X., Zimmerman, E. R., Narayan, R., \& McClintock, J. E. 2005, ApJS, 157,335

Massey, P., Johnson, K. E., \& Degioia-Eastwood, K. 1995, ApJ, 454, 151

Menten, K. M., Reid, M. J., Forbrich, J., \& Brunthaler, A. 2007, A\&A, 474, 515

Miller-Jones, J. C. A., Jonker, P. G., Dhawan, V., et al. 2009, ApJ, 706, L230

Mirabel, I. F., \& Rodrigues, I. 2003, Science, 300, 1119

Orosz, J. A., McClintock, J. E., Aufdenberg, J. P., et al. 2011, ApJ, 742, 84 (Paper II)

Orosz, J. A., McClintock, J. E., Narayan, R., et al. 2007, Nature, 449, 872

Reid, M. J. 1993, ARA\&A, 31, 345

Reid, M. J., \& Brunthaler, A. 2004, ApJ, 616, 872

Reid, M. J., Menten, K. M., Brunthaler, A., et al. 2009a, ApJ, 693, 397

Reid, M. J., Menten, K. M., Zheng, X. W., et al. 2009b, ApJ, 700, 137

Schönrich, R., Binney, J., \& Dehnen, W. 2010, MNRAS, 403, 1829

Stirling, A. M., Spencer, R. E., de la Force, C. J., et al. 2001, MNRAS, 327, 1273

Walker, C., \& Chatterjee, S. 2000, VLBA Scientific Memo 23, http://www.nrao.edu/memos/sci/gps_ion.html

Webster, B. L., \& Murdin, P. 1972, Nature, 235, 37

Xiang, J., Lee, J. C., Nowak, M. A., \& Wilms, J. 2011, ApJ, 738, 78

Ziolkowski, J. 2005, MNRAS, 358, 851 